\title{
ON CUBIC TRANSFORMATIONS OF ORTHOGONAL POLYNOMIALS
}

\section{PIERRE BARRUCAND AND DAVID DICKINSON}

After investigating generalizations of the quadratic transformation relating Hermite and Laguerre polynomials

$$
H_{2 n}(x)=(-1)^{n} 2^{2 n} n ! L_{n}^{(-1 / 2)}\left(x^{2}\right),
$$

T. S. Chihara asked [1] for an example of a pair of orthogonal polynomial sets $\left\{R_{n}(x)\right\}$ and $\left\{S_{n}(x)\right\}$ such that $R_{3 n}(x)=S_{n}(z)$ where $z$ is a cubic in $x$. It is the purpose of this paper to exhibit such examples.

Let $\left\{S_{n}(z)\right\}$ be a set of monic polynomials with $S_{n}(-z)$ $=(-1)^{n} S_{n}(z)$. For this set to be a set of orthogonal polynomials, it is both necessary and sufficient (Shohat [3, pp. 454 and 456]) that there exist a sequence of positive numbers $\left\{B_{n}\right\}$ such that

$$
z S_{n}(z)=S_{n+1}(z)+B_{n} S_{n-1}(z), \quad n=1,2, \cdots,
$$

or equivalently,

$$
\begin{array}{r}
\left(x^{3}+\beta x\right) S_{n}\left(x^{3}+\beta x\right)=S_{n+1}\left(x^{3}+\beta x\right)+B_{n} S_{n-1}\left(x^{3}+\beta x\right), \\
n=1,2, \cdots .
\end{array}
$$

We look for a monic set of orthogonal polynomials $\left\{R_{n}(x)\right\}$ with $R_{n}(-x)=(-1)^{n} R_{n}(x)$ and with a subset $\left\{R_{3 n}(x)\right\}$ that satisfies a recurrence relation with the same coefficients,

$$
\left(x^{3}+\beta x\right) R_{3 n}(x)=R_{3 n+3}(x)+B_{n} R_{3 n-3}(x), \quad n=1,2, \cdots .
$$

Such a set of orthogonal polynomials exists if (3) can be formed by iterating

$$
x R_{n}(x)=R_{n+1}(x)+b_{n} R_{n-1}(x), \quad n=1,2, \cdots,
$$

the condition (with $b_{n}>0, n=1,2, \cdots$ ) that is equivalent to the condition that $\left\{R_{n}(x)\right\}$ is an orthogonal set.

Iterating (4), we may write successively

$$
\begin{aligned}
x R_{3 n}(x) & =R_{3 n+1}(x)+b_{3 n} R_{3 n-1}(x), \\
x^{2} R_{3 n}(x) & =\left(R_{3 n+2}(x)+b_{3 n+1} R_{3 n}(x)\right)+b_{3 n}\left(R_{3 n}(x)+b_{3 n-1} R_{3 n-2}(x)\right),
\end{aligned}
$$

Received by the editors February 21, 1966. 


$$
\begin{aligned}
x^{3} R_{3 n}(x)= & \left(R_{3 n+3}(x)+b_{3 n+2} R_{3 n+1}(x)\right) \\
& +\left(b_{3 n+1}+b_{3 n}\right)\left(R_{3 n+1}(x)+b_{3 n} R_{3 n-1}(x)\right) \\
& +b_{3 n} b_{3 n-1}\left(R_{3 n-1}(x)+b_{3 n-2} R_{3 n-3}(x)\right)
\end{aligned}
$$

and thus

$$
\begin{aligned}
\left(x^{3}+\beta x\right) R_{3 n}(x)= & R_{3 n+3}(x) \\
& +\left(b_{3 n+2}+b_{3 n+1}+b_{3 n}+\beta\right) R_{3 n+1}(x) \\
& +\left(b_{3 n+1}+b_{3 n}+b_{3 n-1}+\beta\right) b_{3 n} R_{3 n-1}(x) \\
& +b_{3 n} b_{3 n-1} b_{3 n-2} R_{3 n-3}(x), \quad n=1,2, \cdots
\end{aligned}
$$

is the same recurrence relation as (3) provided $\beta$ and the $b_{n}$ are such that

$$
\begin{aligned}
b_{3 n+2}+b_{3 n+1}+b_{3 n}+\beta & =0, & & n=1,2, \cdots, \\
b_{3 n+1}+b_{3 n}+b_{3 n-1}+\beta & =0, & & n=1,2, \cdots, \\
b_{3 n} b_{3 n-1} b_{3 n-2} & =B_{n}, & & n=1,2, \cdots
\end{aligned}
$$

Thus, assuming these three conditions (5) on the recurrence relations (1) and (4), the polynomial sets $\left\{S_{n}\left(x^{3}+\beta x\right)\right\}$ and $\left\{R_{3 n}(x)\right\}$ satisfy the same recurrence relations $((2)$ or (3)) and if we further place the initial restrictions

$$
S_{0}\left(x^{3}+\beta x\right)=R_{0}(x)
$$

and

$$
S_{1}\left(x^{3}+\beta x\right)=R_{3}(x),
$$

then we will have

$$
S_{n}\left(x^{3}+\beta x\right)=R_{3 n}(x), \quad n=0,1,2, \cdots .
$$

Condition (6) is satisfied because our assumption that polynomial sets are monic implies $S_{0}(z)=R_{0}(x)=1$. Since $S_{1}(z)=z$, condition (7) becomes $x^{3}+\beta x=R_{3}(x)$ or, because $R_{3}(x)=x R_{2}(x)-b_{2} R_{1}(x)=$ $x^{3}-\left(b_{1}+b_{2}\right) x$, we may replace $(7)$ by the condition

$$
b_{1}+b_{2}+\beta=0 \text {. }
$$

Thus, after combining (8) and the three conditions (5), we have established the

THEOREM. If $\left\{S_{n}(z)\right\}$ is a set of orthogonal polynomials with $S_{0}(z)$ $=1, S_{1}(z)=z$ and

$$
z S_{n}(z)=S_{n+1}(z)+B_{n} S_{n-1}(z), \quad n=1,2, \cdots
$$


and if there exist numbers $\beta$ and $b$ and $a$ sequence of positive numbers $\left\{b_{n}\right\}$ such that the four conditions

$$
\begin{aligned}
b_{1}+b+\beta & =0, & & \\
b_{3 n-1} & =b, & & n=1,2, \cdots, \\
b_{3 n+1}+b_{3 n}+b+\beta & =0, & & n=1,2, \cdots, \\
b_{3 n} b b_{3 n-2} & =B_{n}, & & n=1,2, \cdots,
\end{aligned}
$$

are met, then the polynomials $\left\{R_{n}(x)\right\}$ defined by $R_{0}(x)=1, R_{1}(x)=x$, and

$$
x R_{n}(x)=R_{n+1}(x)+b_{n} R_{n-1}(x), \quad n=1,2, \cdots
$$

form an orthogonal set with the property

$$
S_{n}\left(x^{3}+\beta x\right)=R_{3 n}(x), \quad n=0,1, \cdots .
$$

After this theorem, one naturally inquires about expressing the polynomials of the sets $\left\{R_{3 n+1}(x)\right\}$ and $\left\{R_{3 n+2}(x)\right\}$ in terms of the polynomials of the set $\left\{S_{n}\left(x^{3}+\beta x\right)\right\}$. We can express $R_{3 n+1}(x)$ in terms of $R_{3 n}(x)$ and $R_{3 n+3}(x)$ by successively combining recurrence relations:

$$
\begin{aligned}
x R_{3 n+1}(x) & =R_{3 n+2}(x)+b_{3 n+1} R_{3 n}(x), \\
x^{2} R_{3 n+1}(x) & =\left(R_{3 n+3}(x)+b_{3 n+2} R_{3 n+1}(x)\right)+x b_{3 n+1} R_{3 n}(x), \\
\left(x^{2}-b_{3 n+2}\right) R_{3 n+1}(x) & =R_{3 n+3}(x)+x b_{3 n+1} R_{3 n}(x) .
\end{aligned}
$$

Thus we are led to the

CoRollary. For polynomial sets meeting the hypotheses of the theorem,

$$
\begin{array}{r}
R_{3 n+1}(x)=\left(x^{2}-b\right)^{-1}\left(S_{n+1}\left(x^{3}+\beta x\right)+x b_{3 n+1} S_{n}\left(x^{3}+\beta x\right)\right), \\
n=0,1, \cdots, \\
R_{3 n+2}(x)=\left(x^{2}-b\right)^{-1}\left(x S_{n+1}\left(x^{3}+\beta x\right)+b_{3 n+2} b_{3 n+1} S_{n}\left(x^{3}+\beta x\right)\right), \\
n=0,1, \cdots .
\end{array}
$$

A particularly simple set of constants meeting the conditions of the theorem is $b_{n}=B_{n}=1$ for $n>1, \beta=-3$, and $b_{1}=B_{1}=2$. For this choice of constants, the two sets $\left\{R_{n}(x)\right\}$ and $\left\{S_{n}(x)\right\}$ are identical and $R_{3 n}(x)=R_{n}\left(x^{3}-3 x\right), n=0,1, \cdots$. The recurrence relation

$$
\begin{aligned}
& x R_{n}(x)=R_{n+1}(x)+R_{n-1}(x), \quad n=1,2, \cdots, \\
& x R_{1}(x)=R_{1}(x)+2 R_{0}(x)
\end{aligned}
$$


and the polynomials $\left\{R_{n}(x)\right\}$ when transformed by $R_{0}(2 x)=T_{0}(x)$ and $R_{n}(2 x)=2 T_{n}(x), n=1,2, \cdots$, lead us to the nonmonic set $\left\{T_{n}(x)\right\}$ defined by

$$
\begin{aligned}
T_{0}(x) & =1, \quad T_{1}(x)=x, \\
2 x T_{n}(x) & =T_{n+1}(x)+T_{n-1}(x), \quad n=1,2, \cdots .
\end{aligned}
$$

But this is a definition of the Tchebichef polynomials of the first kind $[2,(10.11)]$. Thus we have shown that for the Tchebichef polynomials of the first kind $\left\{T_{n}(x)\right\}$,

$$
\begin{aligned}
& T_{3 n}(x)=T_{n}\left(4 x^{3}-3 x\right), \quad n=0,1, \cdots, \\
& T_{3 n+1}(x)=\left(4 x^{2}-1\right)^{-1}\left(T_{n+1}\left(4 x^{3}-3 x\right)+2 x T_{n}\left(4 x^{3}+3 x\right)\right), \\
& n=0,1, \cdots, \\
& T_{3 n+2}(x)=\left(4 x^{2}-1\right)^{-1}\left(2 x T_{n+1}\left(4 x^{3}-3 x\right)+T_{n}\left(4 x^{3}-3 x\right)\right), \\
& n=0,1, \cdots \text {. }
\end{aligned}
$$

The cubic transformation (10), which may be written as $T_{8 n}(x)$ $=T_{n}\left(T_{3}(x)\right)$, is a special case of the transformation of arbitrary degree

$$
T_{m n}(x)=T_{m}\left(T_{n}(x)\right), \quad m, n=0,1, \cdots,
$$

that is an easy consequence of the definition $T_{n}(\cos \theta)=\cos n \theta$.

For a less trivial example of the theorem, let the $S_{n}(z)$ be the monic Legendre polynomials defined in terms of the ordinary Legendre polynomials $P_{n}(z)$ by $2^{n}(1 / 2)_{n}(n !)^{-1} S_{n}(z)=P_{n}(z), n=0,1, \cdots$, or equivalently, defined directly by

$$
\begin{aligned}
S_{0}(z) & =1, \quad S_{1}(z)=z, \\
x S_{n}(z) & =S_{n+1}(z)+\frac{n^{2}}{(2 n+1)(2 n-1)} S_{n-1}(z), \quad n=1,2, \cdots
\end{aligned}
$$

Here we have

$$
B_{n}=\frac{n^{2}}{(2 n+1)(2 n-1)}, \quad n=1,2, \cdots,
$$

and the remaining numbers of the theorem may be chosen as

$$
\begin{aligned}
b_{3 n} & =\frac{n}{2 n+1}, & n & =1,2, \cdots, \\
b_{3 n+1} & =\frac{n+1}{2 n+1}, & n & =0,1, \cdots,
\end{aligned}
$$




$$
b_{3 n+2}=b=1, \quad \beta=-2, \quad n=0,1, \cdots .
$$

That these polynomials $\left\{R_{n}(x)\right\}$ where

$$
\begin{aligned}
2^{n}(1 / 2)_{n}(n !)^{-1} R_{3 n}(x)= & P_{n}\left(x^{3}-2 x\right), \\
2^{n+1}(1 / 2)_{n+1}((n+1) !)^{-1} R_{3 n+1}(x)= & \left(x^{2}-1\right)^{-1}\left(P_{n+1}\left(x^{3}-2 x\right)\right. \\
& \left.+x P_{n}\left(x^{3}-2 x\right)\right), \\
2^{n+1}(1 / 2)_{n+1}((n+1) !)^{-1} R_{3 n+2}(x)= & \left(x^{2}-1\right)^{-1}\left(x P_{n+1}\left(x^{3}-2 x\right)\right. \\
& \left.+P_{n}\left(x^{3}-2 x\right)\right)
\end{aligned}
$$

are orthogonal over a bounded domain follows from the boundedness of the coefficients in the recurrence relation (4) by a theorem due to Shohat $([3$, p. 458$])$. Since the zeroes of the polynomials of the set $\left\{P_{n}(z)\right\}$ are dense in the domain $|z| \leqq 1$, the zeroes of the polynomials of the set $\left\{P_{n}\left(x^{3}-2 x\right)\right\}$ (which are the same as the zeroes of the set $\left.\left\{R_{3 n}(x)\right\},(n=0,1, \cdots)\right)$ are dense in the domain $D$ consisting of the points $x$ such that $\left|x^{3}-2 x\right| \leqq 1$. This $D$ is the union of the three disjoint intervals $\left(\left(-1-(5)^{1 / 2}\right) / 2,-1\right),\left(\left(1-(5)^{1 / 2}\right) / 2\right.$, $\left.\left(-1+(5)^{1 / 2}\right) / 2\right)$, and $\left(1,\left(1+(5)^{1 / 2}\right) / 2\right)$. It follows from Szegö $([4$, Theorem 6.1.1]) that this domain $D$ is precisely the "true" domain of orthogonality of this set $\left\{R_{n}(x)\right\}$.

\section{BIBLIOGRAPHY}

1. T. S. Chihara, On kernel polynomials and related systems, Boll. Un. Mat. Ital. (3) 19 (1964), 451-459.

2. A. Erdélyi et al., Higher transcendental functions, McGraw-Hill, New York, 1953.

3. J. Shohat, The relation of the classical orthogonal polynomials to the polynomials of Appell, Amer. J. Math. 58 (1936), 453-464.

4. G. Szegö, Orthogonal polynomials, Amer. Math. Soc. Colloq. Pub. Vol. 23, Amer. Math. Soc. Providence, R. I., 1959.

Institut Blaise Pascal and

UNIVERSITY OF MASSACHUSETtS 